\title{
Research on youth violence risk/needs assessments
}

\begin{abstract}
Assessments to identify the probable risk that a young person will commit an act of violence in the future has evolved since the 1990's from unaided clinical judgment to clinical risk and needs tools that inform treatment. The measure of validity has become the ROC which measures the correct classification rate of events which occur in low frequency. A new evolving standard of functionality for risk assessment tools is incorporating recommendations for evidence based treatment into the risk instrument. Research is continuing on two instruments by the author, the CARE2 and the AVRRT. The CARE2 measures the risk of future violence and treatment needs of youth with histories of chronic violence and delinquent behavior. The AVRRT measures the relative risk of violence and treatment needs of young people that are severely psychotic or on the Autism spectrum and delusional, paranoid, socially awkward, narcissistic, not in treatment and facing a significant major life stressor. Research on both instruments will be described, as well as comparisons with similar tools will be provided. The CARE2 has had 2 major studies, one by the author in 2007 and one in 2014 by Drs. Marvin Tossey, Victoria Venable, and Lawrence Becker at Salisbury University. The results indicate that the CARE2 has good reliability and validity which is comparable other risk assessment tools. Additionally the CARE2 uses items weighted by correlation with future violence, gender and age norms, norms for children below the age of 12 , and recommendations for interventions using evidence based and promising practices. The AVRRT has one study by the Author in 2014 establishing a profile for a second distinct groups of violent males
\end{abstract}

Keywords: youth violence, youth violence risk assessment, evidence based practice, risk assessment, youth violence intervention; chronic violence, psychotic violence, autism spectrum violence
Volume 3 Issue I - 2015

Kathryn Seifert
CARE2, LLC, England

Correspondence: Kathryn Seifert, CARE2, LLC, 2336

Goddard Parkway Salisbury, MD 2180I, England,

Email kathrynseifert@drkathrynseifert.com

Received: December 3I, 2014 | Published: June 01, 2015

\section{Background: The History of the Development of Risk Assessment Tools}

There are five well-known and frequently used assessments to identify risk for future violent acts in the youth category. These tools are often used primarily to make decisions for out of community or higher security placements for youth in the juvenile justice system. These are the SAVRY, ${ }^{1}$ LS-CMI,${ }^{2}$ PACT,${ }^{3}$ CARE- $2,{ }^{4}$ and PCL-YV. ${ }^{5}$

Prior to the 1990's risk assessment was done by interview alone and the results of such assessments were not heavily researched as to accuracy. It is now known that unguided clinical judgment of high, medium or low risk of future violence through interview without a thorough multifaceted assessment which includes a knowledge of the commonly known individual, environmental, community, school, and family risk factors for violence were not accurate beyond chance levels. ${ }^{6-8}$

In the early 1990's actuarial tools began to be developed in Canada where the government put large amounts of grant money into developing these tools. Most were standardized on male adult, mentally ill offenders that were in prison or a hospital housing the not criminally responsible. Little work at that time had been done on aggressive women or youth. ${ }^{7}$

The first violence risk tools were based on static factors that could not be changed such as history of violence. They were easy to score and highly reliable in that different raters scored them similarly. ${ }^{6}$ Tools had low $(\mathrm{ROC}=.60-.70)$ to moderate validity. ${ }^{9}$

The next generation of research on such tools added static factors that could be changed with interventions such as problem solving and anger management skills. These were more difficult to score reliably, but implied possible targets for interventions. It was theorized that the more risk factors that a young person had, the higher the risk for future violence. Consequently, it was also theorized that if a score based on risk factors was reduced, the individual's risk of dangerousness was also reduced. Guidance as to treatment modalities was slim or non-existent. ${ }^{69}$ Most tools were adult tools, standardized on a forensic population residing in Psychiatric hospitals. These tools were developed on the theory that there was a single profile of violent males. From this history, youth tools were developed along the same lines as previous adult tools.

The standard accuracy rate of the existing youth violence risk assessment tools in classifying or rating high, medium, or light future violence today can be seen in.(Table 1).

Therefore, agencies should use tools with a predictive validity of $65-80 \%$ that also have reliability standards in an equal or greater range. Tools for youth that have this standard or greater are the SAVRY, CARE-2, PCL-YV (male), PACT and LS-CMI. ${ }^{6,8-11}$ The standardization sample should also match the population upon which the instrument is being used. All of these instruments use very similar risk factors that have been supported by the research literature. A small study demonstrated the correlation of the scores between the CARE-2, the BASC Aggression scale $(r=.92)$, the PCL-YV $(r=.71)$ and the SAVRY $(r=.72){ }^{4}$

Subsequently, Guided Clinical Judgment was developed and espoused, especially for youth, because we expect youth to be able to reduce risk factors and increase resiliency over time with assistance. Additionally, it was also theorized that using group norms to evaluate individuals would overstate the ability of these tools to predict an actual act of violence by a single individual. Therefore guided clinical judgment tools were preferred at that time. These tools used no numeric scoring and did not take advantage of the statistical 
analysis of the data, but allowed clinicians to use clinical judgment guided by known risk factors to make decisions about future risk of dangerousness. ${ }^{1}$ Recommendations by the scores on these tools for evidence based treatment was mostly lacking.

Over time, guided clinical judgment was found to be as accurate as stand-alone actuarial instruments. However, in some cases, subscales of the instruments were more highly correlated with actual future violence than the total score. ${ }^{6}$ This has caused much controversy in the field because these instruments should not ignore items that can help guide treatment, but these items may not be statistically useful in assessing future risk of violence. What has evolved is an integration of clinical and risk and resiliency items that do not have equal predictability. Therefore, some test creators have moved away from a standard scoring system and others have created a weighted system for items based on the strength of the correlation of the item to actual violence. ${ }^{6}$ In using this rationale, tools can classify youth at high, medium or low risk of the presence or absence of future violence, the severity of violence, or the chronicity of future violence. Most tools measure the probability of the presence or absence of future acts of violence. ${ }^{9}$ The CARE2 was created to measure the probable risk of chronic violence based on the theories and research of Piquero ${ }^{12}$ and Moffitt. ${ }^{13}$

Table I Comparison of most commonly used Youth Risk assessment tools

\begin{tabular}{|c|c|c|c|c|c|c|c|c|}
\hline Tool & Author & $\begin{array}{l}\text { Copyright } \\
\text { Date }\end{array}$ & Type of Tool & ROC for Violence & Ages & $\begin{array}{l}\text { Cross } \\
\text { Validation } \\
\text { Studies }\end{array}$ & $\begin{array}{l}\text { Treatment } \\
\text { Plan with } \\
\text { EBP }\end{array}$ & $\begin{array}{l}\text { Case } \\
\text { Management } \\
\text { Tool }\end{array}$ \\
\hline CARE2 & $\begin{array}{l}\text { Seifert K } \\
{[10]}\end{array}$ & $2003-2011$ & Risk and Needs & $\begin{array}{l}0.75-.94 \text { for males and } \\
.67-.97 \text { for females for } \\
\text { chronic violence }\end{array}$ & 19-Jun & Yes & Yes & Yes \\
\hline SAVRY & Borum [I] & 2002 & $\begin{array}{l}\text { Guided Clinical } \\
\text { Judgment }\end{array}$ & $.66-.77$ & 18-Dec & Yes & No & No \\
\hline LS-CMI & $\begin{array}{l}\text { Andrews, } \\
2010[2]\end{array}$ & 2010 & Risk and Needs & $.61-.73$ & 18-Dec & Yes & No & Yes \\
\hline РACT & \multicolumn{2}{|c|}{ Noble Software Group [3] } & Risk and Needs & 0.85 & UNK & UNK & Yes & Yes \\
\hline PCL-YV & Forth [5] & 2003 & $\begin{array}{l}\text { Structured } \\
\text { Interview, } \\
\text { Measures } \\
\text { Psychopathy }\end{array}$ & $\begin{array}{l}.73 \text { males \& } .50 \text { for } \\
\text { females }\end{array}$ & I8-Dec & Yes & No & No \\
\hline $\begin{array}{l}\text { Unaided } \\
\text { Clinical } \\
\text { Judgment }\end{array}$ & NA & NA & $\begin{array}{l}\text { Unaided Clinical } \\
\text { Judgment }\end{array}$ & 0.50 (Chance levels) & NA & No & No & No \\
\hline
\end{tabular}

Table 2 Comparison of CARE2 ROC's (Area Under the Curve) for 3 violence states

\begin{tabular}{|c|c|c|c|}
\hline Males & Present/Absent & Severity & Chronicity \\
\hline Correlation (relationship $0-100$ expressed as a percent) & $.401(p<.001)$ & $.441(p<.001)$ & $.376(p, .001)$ \\
\hline ROC (rate of classifying a person into the correct group - violent/not violent) & 0.744 & 0.758 & 0.745 \\
\hline Females & Present/Absent & Severity & Chronicity \\
\hline Correlation (relationship 0 - 100 expressed as a percent) & $.155(p>.05)$ & $.222(p<.05)$ & $.354(p<.01)$ \\
\hline ROC (rate of classifying a person into the correct group - violent/not violent) & 0.58 & 0.614 & 0.673 \\
\hline
\end{tabular}

Moffit's ${ }^{13}$ and Piquero's ${ }^{12}$ works were used in choosing and weighting risk factors for the CARE-2. Their work showed that for youth there were 2 general trajectories that could be identified in terms of chronic violent behaviors among youth. The national Institute of Justice wrote multiple articles on these topics. ${ }^{14}$ It was hypothesized and supported that there was a lifelong trajectory and a shorter teen trajectory of delinquent behavior that required very different interventions. The short teen trajectory which often begins in the teen years and is over by adulthood responds well to interventions of the traditional kind and has a higher recovery rate. It does not lead to chronic and severe violence. Traditional therapy for the lifelong trajectory offenders has a recovery rate of $30 \%$ or less. Most youth from the life-long trajectory spend their lives in and out of jails and rehabs most of their lives with criminal justice and substance abuse recidivism rates from $60-80 \%$ through adulthood. This body of work indicates that the characteristics of the two groups are different and require different interventions to be effective. The more complex cases have multiple risk factors, few resiliency factors, and problem behaviors begin before the age of 13. This group responds to interventions such as case management, coping skill building, wrap around, intensive services, job skill building and family therapy. ${ }^{15}$
Additionally, the existing youth violence risk assessment tools, SAVRY, LS-CMI, and PCL-YV, can only be used for youth ages 12 and above. Therefore the CARE-2 was normed on youth ages 6-19 to capture the pre-adolescent youth at risk for chronic violence. In this way, youth can be identified for prevention activities.

Recent examination of public mass murder data in the US has identified a second group of violent youth with a profile that is very different from the youth with a lifelong trajectory of chronic violence. These youth are psychotic or on the Autism spectrum and not receiving the support and treatment they need. Characteristics overlap the chronic violence group but also have some unique difference. This group of violent youth are not chronically violent although they may have had less serious emotional outbursts for many years. Most of these youth are socially awkward, paranoid and narcissistic. Like the chronically violent group they lack certain basic coping skills such as self and anger management, problem solving, communication skills, and effective problem solving. An article in Mother Jones by Follman ${ }^{16}$ examined 62 cases of mass murder over the last 30years. Thirty-eight $(61 \%)$ of perpetrators displayed signs of mental illness before the shootings. Forty-three $(69 \%)$ of murderers committed 
suicide or "suicide by cop." Both percentages are far higher than the national average. An article in Time ${ }^{17}$ stated that having Schizophrenia and a substance abuse problem raises the risk of violence 7 fold. The AVRRT by the author was developed to create a risk reduction plan for severely mentally ill youth and those on the Autism spectrum that are at risk for violence. Substance abuse is a risk factor on this instrument.

Because of this and other research, the new direction is that professionals should be engaged in violence risk reduction planning which applies evidence based practice to the unique set of violence risk and resiliency factors that each individual has, thus attempting to reduce the probability that that person will be violent in the future. Additionally, the creation of multidisciplinary teams to complete evaluations for risk assessment and management, treatment modalities, and placement are rapidly becoming the context within which these assessments take place..$^{18,19}$

\section{The present state of the science of youth violence risk assessment tools}

The SAVRY, designed for ages 12 - 18, measures aggression and recidivism. The ROC's indicate a correct classification rate of a low incidence behavior like aggression, of an individual into a risk group. The total score of the SAVRY has an ROC of .70. The risk score of the SAVRY has an ROC of 89. This indicates that using the risk subscale score alone might be best to use in classifying youth into high, medium or low risk groups than the total score. The SAVRY is a guided clinical judgment tool in which a clinician does not use the scoring system. Levels or types of services are not an element of this tool. The SAVRY has peer review, multiple studies and a manual.

Youth Level of Service-Case Management Inventory is for measuring delinquency and violence among youth ages 12-18. The ROC for violence is .61 - .73 among studies that have been completed. It has a case management tool, peer review, multiple studies and a manual. Its strength is in being used for delinquent youth. ${ }^{2}$

The Psychopathic Checklist - Youth Version (PCL-YV) ${ }^{5}$ is for youth ages 12 - 18. It is primarily a measures psychopathy. The application of the concept of psychopathy to youth is very controversial. The ROC for the PCL-YV for teen male violence is .73; for teen female violence the ROC is .50 or chance levels. It has peer review, multiple studies and a manual. It does not have a case management tool. It is only recommended for use in assessing risk of male violence. The Positive Achievement Change Tool, $\mathrm{PACT}^{3}$ is a measure of delinquency recidivism. The ROC for violent recidivism according to the website is .85 . The tool has a case management component. When used with the Noble Software Group software has an evidence based treatment planning tool. Ages are 17 and below.

CARE-2 - Child and Adolescent Risk/Needs Evaluation ${ }^{10}$ was created for youth ages 6-18. The younger age group is included because the most complex case presentations are of youth with lifelong trajectories of delinquency and violence beginning before the age of $13^{12,13}$ and continues throughout a lifetime unless appropriate intervention is provided in the intensity required for highly complex cases and treatment includes families. The CARE2 has age and gender norms. New research by Tossey $\mathrm{M}^{11}$ at Salisbury University is outlined below. According to this research at Salisbury University, ROC's for the CARE2's assessment of chronic teen violence are .75 (male) and .67 (female). ${ }^{11}$ The CARE-2 also provides intervention recommendations based on the work of Lipsey. ${ }^{15} \mathrm{CARE}-2$ has a case management tool, peer review, multiple studies and a manual

\section{Static and dynamic risk factors for youth violence}

Static risk factors for youth violence ${ }^{20}$ are historical factors that occur at some point in time and never change such as number and types of prior offences, trauma history ${ }^{21}$ death of a parent, history of substance abuse or delinquency. ${ }^{22}$ If measured in the same way, their reliability is very high because they are static. Early actuarial tools were based on Static factors and were highly reliable. However, the items did not lend themselves to measuring the effects of treatment. Dynamic risk factors are those that can change over time and can be affected by treatment such as anger management, social and problem solving skills, ${ }^{23}$ family conflict, school success, and aggression toward others. ${ }^{24}$ They are difficult to measure reliably without very clear definitions and training so that the item is scored in the same way by every practitioner. However, if the items are measured reliably, they can be used to measure outcomes of interventions.

Resiliency factors are those that can help a young person use prosocial coping skills rather than aggression ${ }^{25}$ to get his needs met, such as school success, compliance with treatment, pro-social activity with pro-social peers, empathy ${ }^{26-28}$ and pro-social peers. Risk and resiliency factors can involve the characteristics of the youth, or his relationships with family, school, community, and peers. Mental health problems, substance abuse, and trauma can be contributing factors contributing to reactions to stressors and deficiencies in coping skills. The Office of Juvenile Justice and Delinquency Prevention determined that more than 5 risk factors and less than 4 resiliency factors increased the risk of youth violence by $80 \%{ }^{14}$

\section{CARE=2}

The original research on the CARE-2 is outlined in the Manual. ${ }^{4}$ The sample included 1200 youth from juvenile services, mental health, schools, and residential samples from across the US, ages 6 to 19 . The CARE2 contains 44 risk items and 13 resiliency factors. ${ }^{4}$ There are both static and dynamic factors, as well. Categories of risk factors are historical behavior items, historical family items, historical substance abuse and neurological items, and recent mental health, skill, education items. Items are weighted according to their correlation with actual violence in the first study. The chart in the CARE2 indicates scores that are similar to male and female, preadolescent and adolescent youth that are not similar, mildly similar, moderately similar, and highly similar to youth with chronic (greater than 3) assaults using means and standard deviations. None of the youth in the standardization group for "not similar to youth with chronic assaults had committed any assaults at any time. All of the youth in the group that was "highly similar to youth with chronic assaults" had committed more than 3 assaults and were more than 2 standard deviations above the mean for the entire group.

Reliability (alpha $=.82$ ) and convergent validity for chronic violence on the CARE2 (pre-adolescent males ROC $=.86$; Teen males $\mathrm{ROC}=.82$; pre-teen females $\mathrm{ROC}=.91$; teen females $\mathrm{ROC}=$ .86) were established with the original research. Face and convergent validity and reliability were very good.

\section{0 | 4 CARE-2 research}

New research by Dr. Tossey and his team at Salisbury University was funded by MIPS and stage 1 was completed in 2014. The Maryland Industrial Partnerships (MIPS) program is an initiative of the Maryland Technology Enterprise Institute (M. Tech) in the A. James Clark School of Engineering at the University of Maryland. 
The results of that study in chart form are in Table 2. Face validity of the instrument is good. Criterion Validity for males is excellent for presence/absence, severity and chronicity of future violence. Criterion validity is average and similar to other risk assessment tools for female chronic future violence. Test retest reliability for the 2014 research was excellent.

\section{AVRRT 2015 research}

Because there appeared to be a group of violent youth that did not fit the same general profile as the youth that had histories of chronic violence, a new instrument was created, the AVRRT. It was hypothesized that a group of youthful public mass murderers that had no prior history of violence. They appeared to be psychotic or on the autism spectrum with considerable narcissism, low coping skills, low frustration tolerance, social awkwardness, delusions, not engaged in treatment and not having sufficient social support. These characteristics were combined in a checklist and discussed with other professionals that work with this population. There is not yet a literature on this group of public mass murderers to draw from. Therefore this is exploratory and descriptive research. Ages in this group appeared to range from 16 to 40 .

\section{Sample}

The sample included 32 subjects ages 16-40. Sixteen had committed public mass murder and had been featured in the media; 8 had been violent but had not committed murder; 8 had not committed any act of violence. All subjects were male. Fifteen (50\%) of the 30 males were less than 20years old. Twenty-one were Caucasian; 3 were African American; 2 were Russian; 1 was Asian; 2 were unknown ethnicity. Twelve $(75 \%)$ of the mass murderers were considered to be severely mentally ill with psychosis or were on the autism spectrum, while $2(25 \%)$ of the violent youth who had not committed murder were psychotic or on the autism spectrum. None of the non-violent group were psychotic or on the spectrum. Fifteen (94\%) of the Murder group had serious difficulty with intimate relationships; $100 \%$ of the violent group had severe difficulty with intimate relationships; 1 $(12 \%)$ of the non-violent group has serious difficulty with intimate relationships.

\section{Procedures}

Information on the mass murderers was taken from public information in the media using the AVRRT. Information on violent males, but not murderers, and non-violent males were collected from closed files from a Public Facility. Consents had been previously signed and only identification numbers were used in the study.

\section{Measurement}

The AVRRT was developed by the author. It has 20 items. Example items include: has been bullied, lacks social and professional support, narcissistic, more than 3 emotional outbursts, mental illness or autism with co-occurring substance abuse, Not in treatment and not taking medication, paranoid, delusional, major life stressor, and violent communications. Items are scored on a " 0 " to " 3 " scale. Zero = absent, 1 = little or probable, 2 = Moderate or Mostly True, $3=$ Severe or Mostly True. The item scores are then added for a total score. Scores for the sample were tabulated and are reported here. It is a very small sample size. Therefore only descriptive statistics are reported.

\section{Results}

It appears that the AVRRT can distinguish between the violent and non-violent subjects, therefore the group was split into 2 groups:
Violent members with an $\mathrm{n}$ of 24 and non-violent members with an "n" of 8 (Table 3). This does not reflect the distribution in a normal population. Using 3 groups the correlation between group and AVRRT score was $.87(\mathrm{p}<.00)$. The correlation between AVRRT score and 2 groups (violent vs Non-Violent was .90 $(\mathrm{p}<.00)$. This result will likely decrease as the sample size gets larger, but will likely have a correlation similar to other tools in the field that range from .70 and .85 .

Table 3 Collection of the AVRRT data produced the following chart

\begin{tabular}{llll}
\hline & Number & Mean & SD \\
\hline Group & 32 & 34 & 16 \\
Non-Violent & 8 & 10 & 4 \\
Violent & 8 & 38 & 8 \\
Mass Murderers & 16 & 44 & 6 \\
\hline
\end{tabular}

Twenty of $24(83 \%)$ of the total violent group were narcissistic. Fourteen of sixteen $(88 \%)$ of the mass murderers and $3(38 \%)$ of the violent persons needed treatment but were not engaged in treatment. None of the non-violent people needed treatment, but were not in treatment. Twelve $(75 \%)$ of the 16 Mass murdserers were psychotic or on the autism spectrum; 2 (25\%) of the violent (but not murderers) subjects were psychotic or on the spectrum; none of the non-violent subjects were psychotic or on the spectrum.

Seventeen $(71 \%)$ of the combined violent and murderer group had violent communications, while only $2(25 \%)$ of the non-violent group had violent communications. The differences between the groups on interest in weapons was relatively small (murderers $(50 \%)$, violent $(37.5 \%)$, non-violent $(25 \%)$ and may disappear with a larger sample. All of the total violent group had poor pro-social coping skills and none of the non-violent group had poor skills in that area. Fifteen of the $16(94 \%)$ murderers had problems on the job or in school; $6(75 \%)$ of the violent group had such problems; and $1(12.5 \%)$ of the nonviolent group had these problems.

The AVRRT has face validity in that it incorporates the risk factors observed from actual mass murderers. Construct validity was measured using the correlation of AVRRT score with presence/ absence of violence which was $.87-.90(\mathrm{p}<.00)$, depending on grouping. This correlation is inflated due to the small sample size that likely has some selection bias found in small samples of convenience. Therefore, providing an ROC would be premature. The AVRRT has identified an initial list of variables to identify the mass murderer group for a violence risk reduction plan guided by the AVRRT. This can be a prevention strategy when the instrument is used before a young person becomes violent. The goal of this work is harm reduction, which includes case finding and reducing the risk factors and thus the likelihood that a young person will act out violently.

\section{Conclusion}

While there is much work yet to do in this arena, the research on this topic is advancing rapidly. Only half of the AVRRT sample was under 20years old. A much larger sample of young people is needed. It is important to identify youth in need of interventions to reduce the risk that they may be violent in the future. To do so, further research is needed on the risk factors for 2 overlapping but unique groups of violent young males: chronically violent and criminal throughout their lifetime and those that are psychotic or on the spectrum and paranoid, narcissistic, facing an ego wounding event, not in treatment, and with limited skills and supports. Two tools are being researched to address identifying both groups of youth and preparing risk reduction plans as a prevention strategy: the CARE2 and the AVRRT. The concept of 2 distinct groups of violent males has not been established in the literature and needs further research. 


\section{Discussion}

Violent behaviors appear to be very complex with differences based on age, gender, and types of violence (chronicity, severity, and presence or absence). Risk and resiliency factors are also complex and unique for each individual. Risk assessments must become much more sophisticated in the future, identifying types of violence and treatment planning specific to a youth's individual needs and those of his family. Norms for ages $6-12$ for the assessment of the chronically aggressive, life-long trajectory of delinquency and violence are an important addition to the field of risk reduction planning so that prevention can be implemented before the age of 13. Risk and resiliency factors for chronic violence have been well researched. A tool to identify and create risk reduction plans for youth that are psychotic or on the spectrum are also important to develop. The next realm for research in this field is violence risk reduction treatment planning. Much more research is needed. This article presented some initial research on these two tools and description of the risk factors found in both groups. Research on risk reduction plans is ongoing.

\section{Limitations of the study}

The research on youth violence risk tools has a history and comparable tools such as the SAVRY, LS-CMI, and PCL-YV. There is also a literature of evidence based practices for intervention for youth with histories of chronic violence. However, the CARE2 and the AVRRT are the first to try to combine risk assessment with evidence based practice to form a treatment plan to reduce future risk of violence. This is a new endeavor and will need much more research which is ongoing. With the exception of the research at Salisbury University, the research reported here is by a single author that created this tool. Author bias is certainly possible and until there is cross validation the research is suspect. There is very little literature on a group of public mass murderers that are either psychotic or on the spectrum. Therefore, there is little to reference for this article. This makes this more theoretical conjecture in terms of the AVRRT. More research and study is certainly needed in this arena by other scientists.

\section{Acknowledgments}

None.

\section{Conflicts of interest}

Author declares there are no conflicts of interest.

\section{Funding}

None.

\section{References}

1. Borum. Structured assessment of violence risk in youth (SAVRY). University of South Florida, Tampa, Florida, USA. 2002

2. Andrews DA, Hoge RD. Youth Level of Service-Case Management Inventory, MHS. 2010.

3. Noble Software Group. Products. 2014.

4. Seifert K. CARE2: Child and adolescent risk and needs evaluation Manual. Boston, MA: Acanthus. 2007.

5. Forth AK. Psychopathic Checklist - Youth Version. 2003.

6. Bloom $\mathrm{H}$, Webster CD. Essential writings in violence risk assessment and management. Center for Addiction and Mental Health, Toronto, Canada, USA. 2007.
7. Quinsey VL, Harris GT, Rice ME ,et al. Violent offenders: Appraising and managing risk (2nd edn), American Psychological Association, Washington DC, USA. 2005.

8. Thompson C. Comparative evaluation of youth justice risk/needs assessment tools. 2006.

9. Welsh JL, Schmidt F, McKinnon L, et al. A comparative study of adolescent risk assessment instruments: predictive and incremental validity. Assessment . 2008;15(1):104-115.

10. Seifert K. CARE2: Child and Adolescent Risk/Needs Evaluation. (2nd edn), Acanthus, Boston, Massachusetts, USA. 2003.

11. Tossey M. Establishing and Improving the Predictive Ability of the CARE2 Assessment Instrument. College Park, Maryland: MIPS, USA. 2014.

12. Piquero AA. Life-course Criminology: Contemporary and classic readings. Wadsworth, Belmont, California, USA. 2011.

13. Moffitt ET. Adolescent-limited and life-course persistent antisocial behavior: A developmental taxonomy. American Psychological Association Inc., Wadsworth, Ohio, USA. 1993;100(4):674-701.

14. Office of Juvenile Justice and Delinquency Prevention. Guide for Implementing the Comprehensive Strategy for Serious, Violent, and Chronic Juvenile Offenders. National Institute of Justice, Rockville, Maryland, USA. 1995

15. Lipsey MW, Howell JC, Kelly MR, et al. Improving the effectiveness of juvenile justice programs: A new perspective on Evidence based practice. Center for Juvenile Justice Reform, Washington DC, USA. 2010.

16. Follman M. Mass Shootings: May be What We Need Is a Better MentalHealth Policy. Mother Jones. 2012.

17. Szalavitz M. After Aurora, Questions About Mass Murder and Mental Illness. Time. 2012.

18. Kashani JH. Youth violence: Psychosocial risk factors, treatment, prevention, and recommendations. Journal of Emotional and Behavioral Disorders. 1999; 7(4):200-210.

19. Seifert K. Multi-disciplinary Behavioral Health Evaluations. J Psychol Clin Psychiatry. 2014;1(5).

20. Seifert K. Youth Violence: Theory, prevention, and intervention. Springer Publishing Company, New York, USA. 2012.

21. Ellickson PL, McGuigan KA. Early predictors of adolescent violence. Am J Public Health. 2000;90(4):566-572.

22. Frick PJ, Cornell AH, Barry CT, et al. Callous-unemotional traits and conduct problems in the prediction of conduct problem severity, aggression, and self-report of delinquency. J Abnorm Child Psychol 2003;31(4):457-470.

23. Zimmerman PM, Mohr C, Spangler G. Genetic and attachment influences on adolescents' regulation of autonomy and aggressiveness. $J$ Child Psychol Psychiatry. 2009;50(11):1339-1347.

24. van der Kolk BA. The neurobiology of childhood trauma and abuse. Child and Adolescent Psychiatric Clinics of North America. 2003;12:293-317.

25. Tremblay RE, Hartup WW, Archer J. Developmental origins of aggression. Guilford Press, New York, USA. 2005.

26. Litvak-Miller WM, McDougall D, Romney DM. The structure of empathy during middle childhood and its relationship to prosocial behavior. Genet Soc Gen Psychol Monogr. 1997;123(3):303-324.

27. Centers for Disease Control. Centers for Disease Control Aversive Childhood Experiences Study. Centers for Disease Control. 2006.

28. Russell AW. Maryland Court of Appeals Upholds the Frye-Reed "General Acceptance" Test for Admissibility of Expert Testimony. Wilson Elser Publications, USA. 2003. 